\title{
1 The Principle of the Common Cause: Its Shapes and Content
}

\subsection{Probability: the Basics}

Before we state the various forms of the Principle, some of which will be of a formal nature, a few definitions are in order.

Definition 1 [Probability space] A probability space is a triple $\langle\Omega, \mathcal{F}, P\rangle$ such that:

- $\Omega$ is a nonempty set;

- $\mathcal{F}$ is a nonempty family of subsets of $\Omega$ which is closed under complement and countable union;

- $\quad P$ is a function from $\mathcal{F}$ to $[0,1] \subset \mathbb{R}$ such that

- $P(\Omega)=1$;

- $\quad P$ is countably additive: for a countable family $\mathcal{G}$ of pairwise disjoint members of $\mathcal{F}, P(\cup \mathcal{G})=\sum_{A \in \mathcal{G}} P(A)$.

In the context of a probability space $\langle\Omega, \mathcal{F}, P\rangle, \Omega$ is called the sample space, $\mathcal{F}$ is called the event algebra, and $P$ is called the probability function (or measure). The members of $\mathcal{F}$ are called events.

The above definition captures the content of the concept of a classical probability space. In one of the chapters to come we will also discuss non-classical spaces, but let us postpone their definition till then. Also, in a later chapter we will treat probability spaces as pairs consisting of a Boolean algebra and a measure defined on it; this is because we will be speaking mostly about finite structures for which any Boolean algebra of subsets is of course complete with regard to the operations of complement and countable union (and vice versa, any such family is a Boolean algebra). In general, though, it may be that a Boolean algebra of subsets of a given set is incomplete with respect to (w.r.t.) the operation of countable union.

The complement of an event $A, \mathcal{F} \backslash A$, will be written as " $A^{\perp}$ ". If it is evident from the context that $A$ and $B$ are events, we will sometimes write " $P(A B)$ " instead of " $P(A \wedge$ $B)$ " or " $P(A \cap B)$ " for "the probability that both $A$ and $B$ occur".

Every event $B \in \mathcal{F}$ such that $P(B) \neq 0$ determines a measure $P_{B}$ on the same event algebra: namely, for any $A \in \mathcal{F}, P_{B}(A):=\frac{P(A B)}{P(B)}$. We define the conditional probability of $A$ given $B$ to be equal to $P_{B}(A)$; to refer to it, we will almost exclusively use the traditional notation " $P(A \mid B)$ ". If $P(B)=0$, we take $P(A \mid B)$ to be undefined.

We will now define the concept of a random variable. Our main reference is Feller (1968), but the formulation of some definitions is inspired by Forster (1988). Since in what follows we do not use continuous random variables, we can omit the usual 
measure-theoretic definitions; in fact, we will only need random variables with a finite number of possible values. This is why by "random variable" we will mean what is traditionally referred to as "finite-valued random variable". In what follows, $X^{-1}(v)$ is the preimage of $v$ under the function $X$.

Definition 2 [Random variable] Let $\langle\Omega, \mathcal{F}, P\rangle$ be a probability space. Let $V$ be a finite subset of $\mathbb{R}$. A random variable on $\Omega$ is a function $X: \Omega \rightarrow V$ such that

$$
\forall v \in V \quad X^{-1}(v) \in \mathcal{F} .
$$

If $|V|=2, X$ is called a binary random variable.

Thus every random variable determines a set of events directly tied to its values. The expression " $P(X=v)$ ", "probability that the random variable $X$ takes the value $v$ ", is to be understood as " $P\left(X^{-1}(v)\right)$ "; this is straightforwardly generalized for any subset of $V$, so that for any $V^{\prime} \subseteq V, P\left(X \in V^{\prime}\right)=\sum_{v \in V^{\prime}} P(X=v)$.

Though random variables as defined above are real-valued functions, on some occasions it might of course be useful to think of them as functions with values of a different type, e.g. expressions "yes" or "no". In numerical contexts below we will always treat binary random variables as if they assume values 0 and 1 .

It is immediate that a random variable $X: \Omega \rightarrow V$ can be thought of as a method of dividing the sample space $\Omega$ into at most $|V|$ pieces-the preimages of the members of $V$. There are two intuitive and important ways of thinking about this, depending on our view of the sample space.

First, $\Omega$ can be considered to consist of all possible outcomes of an experiment-for example, if the experiment is a single toss of a six-sided die, then $\Omega=$ $\{1,2,3,4,5,6\}$. A random variable may correspond to a feature which some outcomes possess; for example, if $X(1)=X(3)=X(5)=$ "yes", and $X(2)=X(4)=X(6)=$ "no", then the feature might be "being odd", and " $P(X=$ " $y e s$ ")" is to be interpreted as "the probability that the outcome of the toss is odd".

On the other hand, sometimes $\Omega$ is to be viewed not as a set of outcomes of an experiment, but rather as the population on which an experiment is conducted. Suppose a group of people is tested for a virus. $\Omega$ will then consist of the test subjects, and $P(X=$ "yes") will mean "the probability that a randomly chosen test subject has the virus”.

Notice also the close correspondence of events and binary random variables. An event $A$ is a subset of the sample space; we can construct a binary random variable so that the preimage of "yes" is $A$ and the preimage of "no" is $A^{\perp}$. Similarly, any binary random variable gives rise (by way of the preimages of its values) to two events, $A$ and $A^{\perp}$.

The concept of "correlation" usually concerns random variables, but in the literature around the Principle of the Common Cause it has frequently been defined for 
event, too. ${ }^{1}$ (Usually no probability spaces are defined and the notion of event is an intuitive one, frequently that of a space-time region.) Since in the course of this work we will mostly be talking about events, and not random variables, we shall continue that practice and begin with the simpler concept.

Definition 3 [Correlation (events)] Let $\langle\Omega, \mathcal{F}, P\rangle$ be a probability space and let $A$, $B \in \mathcal{F}$. We say that $A$ and $B$ are:

- positively correlated, or just correlated, whenever $P(A B)>P(A) P(B)$;

- negatively correlated, or anti-correlated, whenever $P(A B)<P(A) P(B)$;

- uncorrelated, or (probabilistically) independent, whenever $P(A B)=P(A) P(B)$.

To define correlation for random variables, we need the notion of covariance and, for that, the notion of expected value.

Definition 4 [Correlation (variables)] Let $\langle\Omega, \mathcal{F}, P\rangle$ be a probability space and $X$ : $\Omega \rightarrow V, Y: \Omega \rightarrow W$ be random variables on $\Omega$.

- $\quad$ The expected value of $X$ is defined as

$$
E(X):=\sum_{v \in V} v \cdot P(v) .^{2}
$$

- $\quad$ The covariance of $X$ and $Y$ is defined as

$$
\operatorname{Cov}(X, Y):=E((X-E(X)) \cdot(Y-E(Y)))=E(X Y)-E(X) E(Y)
$$

- $\quad$ The correlation coefficient of $X$ and $Y$ is defined as

$$
\rho(X, Y)=\frac{\operatorname{Cov}(X, Y)}{\sqrt{\operatorname{Cov}(X, X)} \cdot \sqrt{\operatorname{Cov}(Y, Y)}} .
$$

The correlation coefficient is not defined if $\operatorname{Cov}(X, X)$ or $\operatorname{Cov}(Y, Y)$ is equal to 0 . We will say the variables $X$ and $Y$ are correlated whenever $\rho(X, Y)>0$.

In the context of the Principle of the Common Cause, what demands explanation is a correlation between events or a dependence between random variables. As for the

1 The relation between the two notions is described e.g. in Forster (1988).

2 A mathematically informed Reader may well expect that we add here something like "whenever the right-hand side of the expression converges; we then say that $X$ has a finite expectation"; similarly, that we should prefix the next two clauses of the definition with "Suppose $X$ and $Y$ have finite expectations”. But notice that by definition we are dealing here with finite random variables only and so no question of convergence arises. 
latter, some recent authors (e.g. Reiss (2007)) say simply that "Two variables $X$ and $Y$ are probabilistically dependent just in case $P(X Y) \neq P(X) P(Y)$." Let us expand this into a definition.

Definition 5 [Dependence (variables)] Let $\langle\Omega, \mathcal{F}, P\rangle$ be a probability space and let $X: \Omega \rightarrow V$ and $Y: \Omega \rightarrow W$ be two random variables on $\Omega . X$ and $Y$ are dependent if

$$
\exists V^{\prime} \subseteq V \exists W^{\prime} \subseteq W: P\left(X \in V^{\prime} \wedge Y \in W^{\prime}\right) \neq P\left(X \in V^{\prime}\right) P\left(Y \in W^{\prime}\right) .
$$

Note that for random variables the concepts of independence and noncorrelation diverge. If two variables are independent, their correlation coefficient is 0 , but not always vice versa; for examples see Feller (1968), p. 236. Still, to restate the above, a non-zero correlation coefficient means the variables are dependent.

For binary variables $X$ and $Y$ their covariance is obviously equal to

$$
P(X=1 \wedge Y=1)-P(X=1) P(Y=1) .
$$

This explains why definition 3 can be seen as a special case of definition 4; events are correlated whenever their corresponding binary variables are, and vice versa.

$A$ note about the pair notation. When we want to refer to the ordered pair containing $A$ and $B$, we use the expression " $\langle A, B\rangle$ ”. Sometimes what we need is just the two-element set $\{A, B\}$; we frequently also refer to such sets as "pairs". The meaning is always evident given the context.

\subsubsection{Screening Off}

Perhaps the most important notion concerning the idea of common causes is one of screening off.

Definition 6 [Screening off] Assume a probability space $\langle\Omega, \mathcal{F}, P\rangle$ is given. Let $A, B \in$ $\mathcal{F}$. An event $C$ is said to be a screener-off for the pair $\{A, B\}$ if

$$
P(A B \mid C)=P(A \mid C) P(B \mid C) .
$$

In the case where $A$ and $B$ are correlated we also say that $C$ screens off the correlation.

If $C$ is a screener-off for $\{A, B\}$, we will also frequently say that $C$ "screens off $A$ from $B$ " and vice versa. Another way of putting the fact is saying that $C$ renders $A$ and $B$ conditionally probabilistically independent. Observe that the screening off condition 1.1 is equivalent to the following:

$$
P(A \mid B C)=P(A \mid C)
$$


provided all the probabilities are defined.

Definition 7 [Statistical relevance] Let $\langle\Omega, \mathcal{F}, P\rangle$ be a probability space. Let $A, B \in \mathcal{F}$. We say that an event $C \in \mathcal{F}$ is positively statistically relevant for $A$ if $P(A \mid C)>P\left(A \mid C^{\perp}\right)$. We say that a family of events $\left\{C_{i}\right\}$ is statistically relevant for $A$ and $B$ if whenever $i \neq j$

$$
\left(P\left(A \mid C_{i}\right)-P\left(A \mid C_{j}\right)\right)\left(P\left(B \mid C_{i}\right)-P\left(B \mid C_{j}\right)\right)>0 .
$$

Notice that $P(A \mid C)>P\left(A \mid C^{\perp}\right)$ is equivalent to $P(A \mid C)>P(A)$, if all the probabilities are defined.

\subsubsection{Observing Probabilities and Correlations}

We now have the requisite definitions of probability and related concepts. But how do we observe probabilities "in the world"? If probabilities are to be "limiting frequencies", as one notable interpretation would have it, then we have a problem, since, as beings capable of only finitely many operations, we naturally observe only relative frequencies in finite samples. We can only pose hypotheses about probabilities-but these hypotheses may be well-grounded, thanks to the law of large numbers (see e.g. Feller (1968), p. 243).

If the probability of our experiment ending with a particular outcome is $\phi$, the more we repeat the experiment, the closer should the observed relative frequency of the outcome come to $\phi$. If the probability is unknown, the question regarding the number of repetitions needing to be conducted for us to be able to offer a reliable hypothesis regarding it is subtle, and the answer to it depends on how reliable we require the hypothesis to be. These issues are treated extensively e.g. in Blalock (1979). The technical details will not be of interest to us; the important thing is that no reliable information about probabilities of particular events (and so, a fortiori, about their correlation, as well as probability distributions and correlation of random variables) can be gathered from a small experimental sample.

It will be worthwhile to reiterate this point in an analysis of single occurrences of events which we find unexpected or surprising. Suppose, for instance, that someone rolled two fair six-sided dice on a flat table and ended up with two sixes. Why are we (a bit) surprised? Is the result improbable? That particular combination (a six on the first die, a six on the second die) is improbable to exactly the same degree $(1 / 36)$ as any other possible combination; so the reason for the surprise must be something different. And perhaps it is two-fold:

1. the sum of the results (12) is maximally different from the expected value (7); and perhaps we implicitly compare the probability of rolling it $(1 / 36)$ with the probability of rolling 11 or more (1/12), 10 or more (1/6) and so on; 
2. all throws end up with the same score, which is quite an improbable event $(1 / 6)$ compared to the alternative, which we implicitly expect to occur.

If $X_{i}$ is the event "die number $i$ ends up with a 6", then $P\left(X_{1} X_{2}\right)=\frac{1}{36}$; but just from the occurrence of that particular event we by no means infer that $P\left(X_{1} X_{2}\right)>P\left(X_{1}\right) P\left(X_{2}\right)$. The reason for the fact that a single occurrence of an improbable coincidence, being a conjunction of other events, startles us, is not that we perceive it as evidence of a yet unsuspected correlation. Otherwise we would always have to accuse lucky dice players, or have pity on unlucky ones, for playing with unfair dice.

This is not to say that a proponent of the frequentist interpretation of probability necessarily cannot speak in any way about probabilities of single events. Reichenbach himself would be an example to the contrary-his way of ascribing probabilities to single events is described in section 72 of Reichenbach (1949). Even though he states on p. 375 that single-case probability is a "pseudo-concept", he develops a way of thinking about the single case as "the limit of the [reference] classes becoming gradually narrower and narrower" (ibid.). However, on his account single-case probabilities are, in contrast to "regular" probabilities, dependent on the state of our knowledge; on the whole, he regards "the statement about the probability of the single case, not as having a meaning of its own, but as an elliptic mode of speech" (ibid., p. 376-377). In any case, a frequentist should not in general let a single occurrence of an event influence his or hers beliefs regarding the probabilities inherent in a given situation.

A different issue is whether the data we are analyzing originates from any sort of probabilistic set-up; whether it is appropriate to consider any underlying probabilities at all. If e.g. some parts of the experiment are influenced by human choice, is it wise to consider the probability of a person choosing a particular option? Cartwright (1999) holds the view that no statements regarding probabilities in the world are true simpliciter, but in fact may only be true ceteris paribus; they need to arise in the context of a "probability machine", a fixed arrangement of components with stable capacities giving rise to regular behaviour.

"We can make sense of the probability of drawing two red balls in a row from an urn of a certain composition with replacement; but we cannot make sense of the probability of six percent inflation in the United Kingdom next year without an implicit reference to a specific social and institutional structure that will serve as the chance set-up that generates this probability" (Cartwright (1999), p. 175). ${ }^{3}$

The chance set-ups may be of various kinds: "the stochastic process is the world line of the persisting object (a die, a socio-economic structure)" (Reiss (2007), emphasis

3 Chapter 7.4 of Cartwright's book contains a detailed description of a probability machine in the context of probabilistic claims about causality made by Salmon (1971). 
ours). With no additional information, though, it is unwise to expect a set of data, and the derived relative frequencies of events, as indicative of probability.

\subsection{The Plurality of the Principles}

The literature on the Principle (henceforth referred to as "PCC") abounds in dissenting opinions regarding its validity. It is "false" (Arntzenius (1992)). It is "non-falsifiable" (Hofer-Szabó et al. (2000)). It is a "fallible epistemic principle" (Reiss (2007)). Lastly, it is "derivable from the second law of thermodynamics"” (Reichenbach (1971)). Since each of the above is well-argued for, and since there is such a plethora of views, the subject clearly must be something different in every case. The Principle some authors are arguing against is not always the same Principle their opponents promote.

The multiplicity of forms of the PCC has already been discussed in the literature in e.g. Berkovitz (2000) and sections 3.4 - 3.5 of Placek (2000), but we shall initially take an approach different from those displayed by these texts. Berkovitz analyzes how the prospects of the Principle depend on which concepts of correlation (between types or tokens) and causation are employed. Placek differentiates various versions of the Principle on the basis of the mathematical constitution of the common causewhether it is a single event or an $n$-tuple of events-and whether it is to explain a single correlation or more. While we will also discuss these important matters later on, right now we propose to consider a gradual process of infusing an initially sketchy and informal Principle with formal content.

Throughout the process we will move from purely "informal” principles to purely "formal" ones. The former may arouse deep intuitions and interesting, yet usually inconclusive discussions; the latter can be formally proved or disproved, but one may doubt their relevance to philosophy, or, in the case an antipathy to all things formal is displayed, to anything interesting at all. This is perhaps the usual case when philosophy meets mathematics: the more formal your considerations, the bigger risk of losing track of significant philosophical content. That said, I have a predilection for formal philosophy, which will perhaps be mostly visible in chapter 5; I find it heartening for a philosopher to be able to prove something from time to time. It would be ideal if an interesting and sound philosophical argumentation could be at least partly based on mathematical proofs.

A side note: probability is a relatively new tool for philosophy. Perhaps a big role in its introduction to philosophy was played by Hans Reichenbach's 1956 book The Direction of Time (to which we refer throughout this essay as "Reichenbach (1971)"), where the PCC was first formulated. Subsequently probability has been widely used by researchers in the field of so called "probabilistic causality". To this day, most philoso-

4 Admittedly, only with an additional assumption. See section 1.3.1. 
phers writing about probability usually simply use expressions like " $P(A)$ ” in contexts in which they would normally say "the probability that $A$ occurs”, without defining any probability spaces. There is a drawback: the notion of event is foggy. The Reader cannot be sure what qualifies as an event and what does not; he or she is expected to rely on his or hers intuitions. We will see an example where this can result in an unfortunate misunderstanding (see p. 21). I believe that philosophy would benefit from every author defining their probability spaces explicitly, at the cost of their texts becoming perhaps a bit more "dry" and the process of writing them getting more unwieldy.

We will not cite any proponents of the Principles listed below, because it seems almost every participant in the discussion uses a Principle which is in at least one small respect different from most of the others.

PCC 1 Suppose there is a correlation between two events, which are not directly causally related. Then there exists a common cause of the correlated events.

Notice that no views on the nature of causality are included in the above formulation. While it is difficult to find authors who would openly advocate this view, some arguments offered against “the” PCC (or “Reichenbach's” PCC)-most notably Soberstyle examples we will discuss in section 1.4.2-actually negate PCC 1, since the probabilistic description of the allegedly existing common cause is largely irrelevant to the argument.

PCC 2 Some correlations demand explanation. Of these, some demand explanation by means of a common cause. In each such case there exists a common cause of the correlated events, which renders them conditionally probabilistically independent.

There are two additions in comparison to PCC 1. First, a qualification is added that perhaps only some (not all) correlations stand in need of common causal explanation; some authors use the word "improbable" to describe them. Second, a probabilistic ingredient is added: the postulated common cause of the correlated events should screen them off.

PCC 3 Let $\langle\Omega, \mathcal{F}, P\rangle$ be a probability space. For any $A, B \in \mathcal{F}$ (such that $\langle A, B\rangle$ belongs to a relation of independence $L_{\text {ind }}$ ), if $P(A B)>P(A) P(B)$, then there exists an event $C \in \mathcal{F}$ (different from both $A$ and $B$ ) such that

$$
\begin{aligned}
P(A B \mid C) & =P(A \mid C) P(B \mid C) ; \\
P\left(A B \mid C^{\perp}\right) & =P\left(A \mid C^{\perp}\right) P\left(B \mid C^{\perp}\right) ; \\
P(A \mid C) & >P\left(A \mid C^{\perp}\right) ; \\
P(B \mid C) & >P\left(B \mid C^{\perp}\right) .
\end{aligned}
$$


This version of the Principle is of a formal nature. The word "cause" is nowhere to be seen; it can be of course introduced, by defining a common cause for $A$ and $B$ as an event meeting the four requirements above. (We assume this definition for the remainder of this section.) PCC 3 is actually meant to possess two variants: with or without the first expression in parentheses. Frequently a relation of independence is introduced; it is usually at least logical independence (so that e.g. the correlation between "heads up" and "tails down" will not stand in need of an explanation in terms of a common cause), and perhaps ideally it is supposed also to cover direct causal independence. However, if $L_{\text {ind }}$ is just logical independence, then PCC 3 is simply false, as it is easy to find examples of spaces with correlations between logically independent events, for which no event meeting the requirements above exists (see e.g. Hofer-Szabó et al. (2000), p. 91). It is also highly unlikely that "fixing" the relation of independence so that it includes less pairs than the relation of purely logical independence will alleviate this difficulty and make the Principle generally plausible. However, an interesting question is: in which classes of probability spaces and for which relations of independence does the Principle hold? We will discuss these issues at length in chapter 5.

To state the last form of the Principle we need to define extension of probability spaces.

Definition 8 [Extension] Let $\mathfrak{A}=\langle\Omega, \mathcal{F}, P\rangle$, be a probability space. A space $\mathfrak{A}^{\prime}=$ $\left\langle\Omega^{\prime}, \mathcal{F}^{\prime}, P^{\prime}\right\rangle$ is called an extension of $\mathfrak{A}$ if there is a Boolean algebra embedding $h$ : $\mathcal{F} \rightarrow \mathcal{F}^{\prime}$ which preserves the measure, that is, $\forall A \in \mathcal{F}, P^{\prime}(h(A))=P(A)$.

PCC 4 Let $\mathfrak{A}=\langle\Omega, \mathcal{F}, P\rangle$ be a probability space. Suppose that $A, B \in \mathcal{F}$ (such that $\langle A, B\rangle$ belongs to a relation of independence $L_{\text {ind }}$ ) are correlated, but there exists no $C \in \mathcal{F}$ (different from both $A$ and $B$ ) such that

$$
\begin{aligned}
P(A B \mid C) & =P(A \mid C) P(B \mid C) ; \\
P\left(A B \mid C^{\perp}\right) & =P\left(A \mid C^{\perp}\right) P\left(B \mid C^{\perp}\right) ; \\
P(A \mid C) & >P\left(A \mid C^{\perp}\right) ; \\
P(B \mid C) & >P\left(B \mid C^{\perp}\right) .
\end{aligned}
$$

Then there exists a space $\mathfrak{A}^{\prime}=\left\langle\Omega^{\prime}, \mathcal{F}^{\prime}, P^{\prime}\right\rangle$ such that $\mathfrak{A}^{\prime}$ is an extension of $\mathfrak{A}$ by means of a homomorphism $h$ and there exists an event $C^{\prime} \in \mathcal{F}^{\prime}$ such that

$$
\begin{aligned}
P^{\prime}\left(h(A) h(B) \mid C^{\prime}\right) & =P^{\prime}\left(h(A) \mid C^{\prime}\right) P^{\prime}\left(h(B) \mid C^{\prime}\right) ; \\
P^{\prime}\left(h(A) h(B) \mid C^{\prime}\right) & =P^{\prime}\left(h(A) \mid C^{\prime}\right) P^{\prime}\left(h(B) \mid C^{\prime}\right) ; \\
P^{\prime}\left(h(A) \mid C^{\prime}\right) & >P^{\prime}\left(h(A) \mid C^{\prime}\right) ; \\
P^{\prime}\left(h(B) \mid C^{\prime}\right) & >P^{\prime}\left(h(B) \mid C^{\prime}\right) .
\end{aligned}
$$

As we have already said, there are numerous counterexamples to PCC 3, which is a statement postulating, for each correlation in a given space, a common cause in 
the same space. PCC 4 is, however, more subtle. Suppose we observe an unexpected correlation during an experiment, but the probability space we have chosen to operate within lacks common causes for the correlated events. But perhaps the choice of the space was unfortunate; perhaps we have not taken some factors into account and a different, more "fine-grained" space, compatible with the observations to the same extent as the original one, provides an explanation for the correlation in terms of a common cause? In other words, can the original space be extended to a space possessing a common cause for the yet unexplained correlation? And in general, is it possible to extend a given probability space to one containing common causes for all correlations? Perhaps surprisingly, the answer to both questions is "yes". We will deal with these matters extensively in chapter 6.

We have already mentioned that the place in which the PCC was introduced was Reichenbach's Direction of Time. Subsequently, regardless of the version of the Principle they are concerned with, many authors credit Reichenbach with the original idea. Some of them (e.g. Hoover (2003), p. 527) content themselves with the following quotation: "If an improbable coincidence has occurred, there must exist a common cause"5. In the next section we will try to convince the Reader that such a selective quotation misses a few important facets of Reichenbach's view of the Principle.

\subsection{What Reichenbach Wrote}

Reichenbach's 1956 book is frequently taken to contain an important metaphysical view of probabilistic causality (see e.g. Williamson (2009)). The main object of the book, however, is to analyze the possibilities of defining time direction by means of causal relations. Part IV discusses the case of macrostatistics and it is there, in chapter 19, where the Principle of the Common Cause originally appears.

Throughout his book Reichenbach frequently writes about probability (his formulas will be put here in modern notation), however, he did not choose to adopt the Kolmogorovian concepts of "event algebra" and "probability space", which were then slowly gaining recognition. The choice was undoubtedly motivated by the fact that he already had his own von Mises-style theory of probability, developed earlier in Reichenbach (1949) (originally issued in German in 1935). It is important to note at the beginning of this section that, for Reichenbach, "the term "probability" is always assumed to mean the limit of a relative frequency" (Reichenbach (1971), p. 123). Therefore the question of probability of an event regarded in isolation of any sequence of its possible occurrences or non-occurrences should be meaningless. To use a popu-

5 Reichenbach (1971), p. 157. 
lar philosophical term, for Reichenbach there should be no such things as single-case probabilities. (But remember the quote referring to an "elliptic mode of speech", p. 6.)

The guiding idea behind Reichenbach's Principle, and the source of-as we will see-an important argument for one of Reichenbach's theses is that "the improbable should be explained in terms of causes, not in terms of effects" (Reichenbach (1971), p. 157); the short version of the Principle of the Common Cause quoted at the end of the previous section comes right at the end of the same paragraph. Let us quote the first examples with which Reichenbach's illustrates his Principle (all quotes from ibid., p. 157):

- "Suppose that lightning starts a brush fire, and that a strong wind blows and spreads the fire, which is thus turned into a major disaster. The coincidence of fire and wind has here a common effect, the burning over of a wide area. But when we ask why this coincidence occurred, we do not refer to the common effect, but look for a common cause. The thunderstorm that produced the lightning also produced the wind, and the improbable coincidence is thus explained."

- "Suppose both lamps in a room go out suddenly. We regard it as improbable that by chance both bulbs burned out at the same time, and look for a burned-out fuse or some other interruption of the common power supply. The improbable coincidence is thus explained as the product of a common cause."

- "Or suppose several actors in a stage play fall ill, showing symptoms of food poisoning. We assume that the poisoned food stems from the same source-for instance, that it was contained in a common meal-and thus look for an explanation of the coincidence in terms of a common cause.”

Keeping in mind the concept of probability quoted above, up to this point it would hardly seem surprising that the Principle-

Reichenbach's PCC-the "coincidence” formulation: "If an improbable coincidence has occurred, there must exist a common cause"

-makes no mention of probability save for the word "improbable" in the antecedent. Reichenbach quickly injects his Principle with more probabilistic content, though. First, he admits that "chance coincidences, of course, are not impossible", since the bulbs may simply burn out at the same moment etc. Therefore, in such cases the existence of a common cause is "not absolutely certain, but only probable" (ibid., emphasis ours), with the probability increasing with the number of repeated coincidences. (Let us just note that the concept of probability implicit here seems to be decidedly epistemic-the more repeated coincidences we observe, the more strongly we should believe in the existence of a common cause-and thus hard to reconcile with the earlier definition.) The author offers another two examples supporting the Principle (ibid., p. 158): 
- "Suppose two geysers which are not far apart spout irregularly, but throw up their columns of water always at the same time. The existence of a subterranean connection of the two geysers with a common reservoir of hot water is then practically certain."

- "The fact that measuring instruments such as barometers always show the same indication if they are not too far apart, is a consequence of the existence of a common cause-here, the air pressure."

We are then advised to "treat the principle of the common cause as a statistical problem" (ibid.). In Reichenbach's view this means that we should assume events $A$ and $B$ have been observed to occur frequently, which enables us to consider probabilities $P(A), P(B)$ and $P(A B)$. The relationship between two (improbably) simultaneously occurring events and both their common cause and effect is depicted in terms of forks seen in figure 1.1.

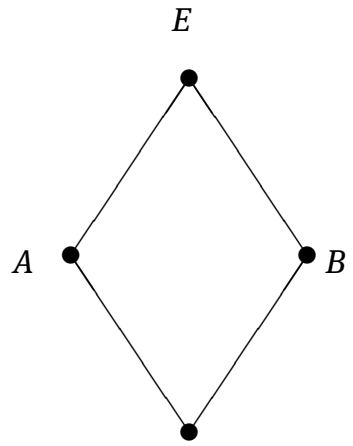

C

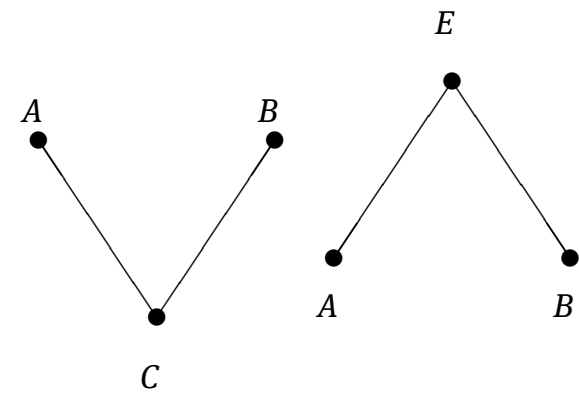

Figure 1.1: A double fork, a fork open towards the future, a fork open towards the past. " $C$ " denotes a cause, " $E$ " denotes an effect.

Reichenbach claims the forks depict statistical relationships between the events. However, in his examples cited above there always is some physical process behind each arrow on the diagram.

The coincidence of events $A$ and $B$ has, for Reichenbach, "a probability exceeding that of a chance coincidence" (ibid., p. 159) precisely when the two events are correlated in terms of our definition 3. Suppose, then, the events are correlated. We "assume that there exists a common cause $C$. If there is more than one possible kind of common cause, $C$ may represent the disjunction of these causes" (ibid.). An important assumption is now that the fork $A C B$ satisfies exactly the statistical requirements listed above 
in the formulation of PCC 3:

$$
\begin{aligned}
P(A B \mid C) & =P(A \mid C) P(B \mid C) ; \\
P\left(A B \mid C^{\perp}\right) & =P\left(A \mid C^{\perp}\right) P\left(B \mid C^{\perp}\right) ; \\
P(A \mid C) & >P\left(A \mid C^{\perp}\right) ; \\
P(B \mid C) & >P\left(B \mid C^{\perp}\right) .
\end{aligned}
$$

Namely, both $C$ and $C^{\perp}$ should screen off $A$ from $B$, and $C$ should be statistically relevant both for $A$ and $B$.

Reichenbach proceeds to point out two explanatory features of the proposed common causes. The first one is that from the conditions (1.3)-(1.6) the correlation between $A$ and $B$ is deducible. (We shall investigate this and related ideas in section 2.1.) This fact is interpreted by Reichenbach as meaning that the fork $A C B$ "makes the conjunction of the two events $A$ and $B$ more frequent than it would be for independent events" (ibid.), and that is why the author proposes to call such forks conjunctive forks. The second explanatory feature of common causes is that, due to screening off, the correlation in a sense "disappears"- "relative to the cause $C$ the events $A$ and $B$ are mutually independent” (ibid.). Due to these features, a common cause makes it possible to derive statistical dependence from an independence. The common cause is therefore the "connecting link", and the conjunctive fork "is therefore the statistical model of the relationship formulated in the principle of the common cause" (ibid., p. 160).

What follows next is the proof of the above mentioned fact that from conditions (1.3)-(1.6) one can derive the correlation between $A$ and $B$. It is thus quite puzzling why, on the next page (163), Reichenbach writes "These results may be summarized in terms of the principle of the common cause (...)”. Which results? So far, the existence of common causes as the middle links in conjunctive forks was distinctively assumed, not reached as any sort of result. What is more important now, though, since the author attempts a justification of the Principle later on, is its formulation (reworded so it would not refer to equations in Reichenbach's text by their numbers):

Reichenbach's PCC-the "correlation" formulation: "If coincidences of two events $A$ and $B$ occur more frequently than would correspond to their independent occurrence, that is, if the events are correlated, then there exists a common cause $C$ for these events such that the fork $A C B$ is conjunctive."

Notice that with the move from speaking about single coincidences to correlations the word "improbable" disappears. In the above formulation there is no division between "probable" and "improbable" (or "unexpected" / "accidental") correlations. Common causes are to exist for all correlations.

What is, for Reichenbach, the relationship between the statistical conditions (1.3)(1.6) and the concept of common cause? Being a common cause of $A$ and $B$ is not sufficient for being the middle link of a conjunctive fork: $A$ and $B$ may simply not be correlated (Reichenbach's example is that of two dice being thrown by the same hand). In 
the other direction, being the middle element of a conjunctive fork is for Reichenbach certainly not sufficient for being a common cause, since common effects may also satisfy conditions (1.3)-(1.6). However, for his idea for defining time direction to work, it is absolutely crucial to ascertain that if a conjunctive fork $A C B$ is open ${ }^{6}$, then $C$ is a common cause of $A$ and $B$ and not their effect. In this way he will be able to frame his definition of time direction in terms of macrostatistics as "In a conjunctive fork $A C B$ which is open on one side, $C$ is earlier than $A$ or $B$ ” (ibid., p. 162). But does he succeed in showing the causal asymmetry of conjunctive forks? This may initially seem to be a side issue for the Principle of the common cause, but it is not: an example of a conjunctive fork open to one side, containing two events and their common effect, such that there is no common cause for the two events which together with them constitutes a conjunctive fork, would be a counterexample to the Principle. The fact that the issue was discussed in this context by perhaps the staunchest proponent of the principle, Wesley Salmon (1984), is another reason for which we will return to it in one of the coming sections.

\subsubsection{Reichenbach's Argument for the Principle}

What is, then, the justification given by Reichenbach for his Principle? It is supposed to follow from the second law of thermodynamics-the entropy of an isolated system which is not in equilibrium tends to increase-supplemented with an additional assumption, labeled “branch hypothesis”, which we shall now consider.

As we said earlier, in his book Reichenbach does not use the formalism of probability spaces which has since then become the standard approach. Instead in $\S 12$ he introduces the so called "Probability Lattice". In the context of the book, it is a mathematical construction for describing processes of mixture. A probability lattice is a two-dimensional matrix; each row represents the history of a single object, e.g. a molecule of gas (thus it is also called a "time ensemble"), and each column is a time-slice through the system under consideration, containing information about the state of all molecules in the system at a given time (being thus also called a "space ensemble"). To use Reichenbach's own example, consider a container with two compartments $-L$ and $R$-and assume there are molecules of nitrogen in compartment $L$ and oxygen in compartment $R$. Suppose the wall dividing the compartments is removed and the substances begin to mix with each other. If we restrict our attention to nitrogen only, and record only the positions of the molecules (in a binary way, " $L$ "

6 This seems to mean that one of two possibilities (one of which is almost immediately excluded) occurs: either (1) $C$ is a common cause of $A$ and $B$, and there exists no common effect $D$ of $A$ and $B$ such that $A D B$ would constitute a conjunctive fork, or (2) $C$ is a common effect of $A$ and $B$, and there exists no common cause $D$ of $A$ and $B$ such that $A D B$ would constitute a conjunctive fork. 
or " $R$ "), the first column of our probability lattice should be filled exclusively with $L s$, while the farther we go to the right, the more the proportion of $L s$ and $R s$ in a given column approaches $1 / 2$.

The lattice will be a "lattice of mixture" (Reichenbach (1971), p. 103) only if it meets a few conditions, discussed on pages 100-103 of the book. The two simpler ones regard the initial column (which should be ordered ${ }^{7}$ ) and the aftereffect in rows (an $R$ at position $i$ in a row increases the chance for an $R$ at position $i+1$ in the same row). The other two, though, "independence of rows" and (especially) "lattice invariance" (which allows making inferences "from the time ensemble to the space ensemble"), are highly non-trivial. It would not be proper to study the conditions here in detail, since they are not at the heart of Reichenbach's argument for the PCC. ${ }^{8}$

The formalism was needed because the branch hypothesis itself refers to a lattice of mixture (Reichenbach (1971), p. 156). The general idea is that the universe, as a whole, is a system with currently low entropy but which increases over time (barring some short-term anomalies). From the main system, smaller systems branch off, and are isolated for a certain period-but they are connected with the main system at both ends. The entropy of these "branch systems" is also (in general) low at one of these points and high at the other; the crucial thing is that the direction towards higher entropy is in general parallel throughout the branch systems. This covers four out of five assumptions making up the hypothesis; the remaining one is that the lattice of branch systems is a lattice of mixture.

Suppose, for now, the branch hypothesis is true. How should the PCC follow? Reichenbach first tries to show (pp. 164-165) how a specific type of ensemble gives rise to a conjunctive fork, transcribed into his lattice notation. This ensemble containst two types of branch systems, $T_{A}$ and $T_{B}$ (systems of the first type may assume state $A$ and the others state $B$ ), such that sometimes a system of type $T_{A}$ and another of type $T_{B}$ coincide in their first state (call it $C$ ). A composite probability lattice is constructed from the two lattices for two system types by appropriately "gluing" some rows on top of others so that for any case of the above mentioned coincidence the row for the system of type $T_{A}$ is on top of the row for the system of type $T_{B}$. For future reference, let us

\footnotetext{
7 In the sense that it should illustrate a state of order; just like in the previous example, the initialordered-state of the system is illustrated by a column with the letter $L$ in all entries.

8 But it has to be noted that while there may be some intuitive appeal of those two conditions being connected with a mixing process, the author himself struggles with his own notation, being forced to use sub-subscripts, and we hope the Reader who consults the book will agree that it is not evident that Reichenbach's formulas in his lattice-lingo adequately express what he says in English. (For example, why does the right-hand side of formula (17) (p. 101) express any “vertical probability” (as defined on p. 99) at all?) Even if these difficulties were dispensed with, there is no justification for lattice invariance save for a reference to Reichenbach (1949), where it is stated that the kinetic theory of gases makes a similar assumption (p. 174).
} 
state that his goal here was to ascertain that "whenever two causal lines leading to $A$ and $B$ are connected by their first element $C$, the fork $A C B$ is conjunctive" ( $\left.{ }^{\star}\right)$.

Then Reichenbach claims that "the branch hypothesis tells us that if a state occurs more frequently in the space ensemble than corresponds to a certain standard, namely, to its probability in the time ensemble, there must have existed an interac-


quantifiers over columns and rows. Should we read it as "the branch hypothesis tells us that if in a lattice of mixture there exist row $k$ and column $i$ such that a certain state occurs more frequently in $k$ than in $i$, (...)" or "the branch hypothesis tells us that if in a given lattice of mixture it is true that for any row $k$ and column $i$ a certain state occurs more frequently in $k$ than in $i,(.$.$) "? The fact that in the previous paragraph we$ seem to have been actually considering three-dimensional probability lattices ${ }^{9}$ does not help, either.

But let us, again, drop this issue (and the issue of whether the above actually follows from the branch hypothesis). The next step in Reichenbach's reasoning is that

"If the two causal lines leading to $A$ and $B$ were not connected by their first elements, the probability of the joint occurrence would be given by $\left.P(A) \cdot P(B) .{ }^{10}{ }^{(\star \star \star}\right)$

This, unfortunately, begs the question. By contraposition and combination with ${ }^{\star}$ ) we get: "if $A$ and $B$ are correlated or anti-correlated, the two causal lines leading to $A$ and $B$ are connected by their first element $C$, and the fork $A C B$ is conjunctive ", which is at first sight an even stronger statement than the PCC. ${ }^{11}$ Statement $\left({ }^{\star \star \star}\right)$ needs to be backed up, but is not. It cannot be backed up by $(\star \star)$-because, however we understand it, it is an implication from the fact that the probability of a given state in the space ensemble is different (higher) than its probability in the time ensemble, without reference to the actual values of the probabilities! So, a priori, it is consistent with ( $\left.{ }^{\star \star}\right)$ that for some $A$ and $B$, the causal lines leading to $A$ and $B$ are not connected by their first elements, but the probability of the joint occurrence is given by $P(A)$. $P(B)+0.05$, which is inconsistent with $\left({ }^{\star \star \star}\right) .{ }^{12}$ Sadly, we have to conclude the argument

9 The additional dimension, apart from rows and columns, stems from the fact that rows from the lattice for systems of type $T_{A}$ are "above" the ones for systems of type $T_{B}$; it cannot be the twodimensional sort of "above" used in statements like "on this very page, the previous line is above this one", since were it so it wouldn't be possible for $A$ s and $B$ s to happen in the same row of the composite lattice, which is explicitly required by Reichenbach's mathematical formulas.

10 ibid.

11 Only at first sight, because if events $A$ and $B$ are anti-correlated, then $A$ and $B^{\perp}$ are positively correlated (and vice versa), so the PCC may also be read as demanding explanation for anti-correlations. 12 The point will be perhaps more palatable if made colloquially: everyone remembers that "correlation does not mean causation". But (since $A$ and $B$, belonging by assumption to isolated branch systems, cannot cause one another) $\left.{ }^{\star \star \star}\right)$ says basically that "absence of causation means absence of correlation”! The author, when claiming $\left.{ }^{\star \star \star \star}\right)$, has to have in mind something similar to the negation 
given by Reichenbach misses a link without which part $\left(^{\star \star \star}\right)$ assumes the thesis. Thus the status of the Principle of the common cause in The Direction of Time is still that of a hypothesis. It does not change the fact that it may well be a valuable rule of human reasoning; simply, Reichenbach does not succeed in showing it to be a well-proved theorem.

It has to be added that Reichenbach himself thought that the PCC "reiterates the very principle which expresses the nucleus of the hypothesis of the branch structure" (ibid., p. 167). Perhaps, then, no separate argument for the PCC is needed and a justification of the hypothesis of the branch structure would suffice. We will argue in the next section that this prospect is sadly also not hopeful, among a few other drawbacks of Reichenbach's account.

\subsubsection{Other Problems with Reichenbach's Approach}

\subsubsection{The Hypothesis of the Branch Structure: “Main System" and Entropy}

The first worry regarding the hypothesis concerns what it is that is supposed to branch. Reichenbach offers a few illustrations. In the first one (fig. 20 from Reichenbach (1971), p. 119) we are supposed to see a "long upgrade of the entropy curve of the universe" and "systems branching off from this upgrade, assuming that these branch systems remain isolated for an infinite time" (ibid., p. 118). The second one (fig. 21 from Reichenbach (1971), p. 127)) differs in that the systems which branch off from the main system return to it and that it contains also a downgrade of the entropy curve.

In both images the vertical axis is supposed to depict entropy. And the problem is that, while not all concepts of entropy are that of an additive quality (see e.g. Palm (1978)), the types of entropy considered by Reichenbach are additive, as he says himself on p. 53 ("If two systems are brought together, their entropies are additive"). Therefore, suppose the universe consisted of a system which from time to time divides into two systems that remain isolated for a certain period and then connect again. It seems to me that Reichenbach's fig. 21 is incompatible with additivity of entropywhen the two systems reconnect, the entropy of the whole is depicted as being equal to the entropy of any of the two, while it should be the sum of the two. A picture which would do justice to the additivity of entropy would not contain a branching curve, though.

Perhaps this is just a pictorial difficulty of no greater import. But the bigger problem with the hypothesis is that it refers to "the main system"; presumably, "the main system of the universe". It is never made clear what the main system is. Is Earth a part

of the hackneyed slogan; namely, that correlation does mean causation, if not between the correlated events (since they occur at the same time or belong to isolated systems), but between them and their common cause. This is yet another informal statement of Reichenbach's PCC. 
of it, or is the humanity in some backwater region of the universe? At first sight, the concept of the main system is important for the hypothesis; the main system is to serve as the root from which the other systems branch, and to which they eventually return. On the other hand, perhaps the hypothesis could be reformulated so that it would refer to an ensemble of systems whose both ending points are in other systems, and which are isolated from all other systems apart from their endpoints. In this case, there would be no distinguished "root", or "main", system-and similarly, there would be no need to use the name "branch system" instead of simply saying "system": all systems would have equal rights, so to speak. One would also have to take care when accommodating the old Assumption 4 ("In the vast majority of branch systems, one end is a low point, the other a high point”) to the new hypothesis; what if a system $K$ branches off a system $L$ at a point of $L$ 's high entropy, but, after a period of isolation, connects with a system $M$ at a point of $M$ 's low entropy? I do not think these difficulties are insurmountable. It is feasible that one could reformulate Reichenbach's hypothesis of the branch structure so that it would not refer to any "main system", while still capturing as much of the intentions of the original author as possible. Then the task of deriving the PCC could be approached again. A problem with this is that one would still be trapped with Reichenbach's probability lattice approach and his notation. We prefer to pursue another option and consider the chances of proving theorems related to the PCC using the machinery of "modern" probability theory. This endeavour is taken up in chapter 5 .

\subsubsection{What do the Initial Examples Illustrate?}

As we said earlier on, Reichenbach himself claims that in his book probability is always to be understood as a limit of a relative frequency. This would seem to preclude ascribing probability to "token", unrepeatable events; in other words, there should be no "single-case probabilities". However, we already quoted passages from Reichenbach (1949) indicating that there is an, albeit elliptic, way of speaking about constructs which are to serve as a substitute for them in Reichenbach's theory. How should we, then, understand the initial examples of common-causal reasoning offered by the author (and quoted here on p. 11), the fire and the wind, the burned-out bulbs, and the ill actors? The common cause is invoked after the occurrence of a single event is observed. At the end of section 1.1.2 we claimed that no beliefs about the probability of such an event should be formed just because of a single occurrence. Reichenbach seems to agree, writing on p. 158, not long after the examples have been presented, “(...) we assume that $A$ and $B$ have been observed frequently; thus it is possible to speak about probabilities $P(A), P(B)$ and $P(B \mid A)(\ldots)$ ". So, in the initial examples we are not supposed to think of probabilities, let alone correlations. Therefore they cannot be of any support for the Principle in its "correlation" formulation; they only illustrate the "coincidence" formulation in action. 
Remember, though, that the two features Reichenbach advertised as due to which a common cause has explanatory value stem from the common cause being a middle link in a conjunctive fork. Since the definition of the fork is probabilistic, if we know nothing about the probability of the given common cause $C$, we cannot judge whether it is the middle link in a conjunctive fork $A C B$, and so cannot benefit from the abovementioned features: (1) that the correlation disappears when the events $A$ and $B$ are considered conditional on $C$, and (2) that the correlation is derivable from the conjunctive fork condition. These two features show us why the PCC may be promoted as one of the principles guiding the human search for explanation, but only in its formulation referring to a "correlation" (p. 13), not in the one bringing up an "improbable coincidence” (p. 11).

In conclusion, Reichenbach's initial examples illustrate only the "coincidence" formulation of the Principle, which lacks the important explanatory features of the "correlation" formulation.

\subsubsection{On Forks Open to the Past}

First let us ask about sufficient conditions for a triple of events $A C B$ to constitute a conjunctive fork. Are the statistical requirements (1.3)-(1.6) enough? Consider some events $A, B$ and their common cause $C$, which operates in a deterministic way: $P(A \mid$ $C)=P(B \mid C)=1, P\left(A \mid C^{\perp}\right)=P\left(B \mid C^{\perp}\right)=0$. Notice that

$$
\begin{aligned}
P(A C \mid B) & =1=P(A \mid B) P(C \mid B) ; \\
P\left(A C \mid B^{\perp}\right) & =0=P\left(A \mid B^{\perp}\right) P\left(C \mid B^{\perp}\right) ; \\
P(A \mid B) & =1>0=P\left(A \mid B^{\perp}\right) ; \\
P(C \mid B) & =1>0=P\left(C \mid B^{\perp}\right),
\end{aligned}
$$

so the triple $A B C$ satisfies the statistical requirements for being a conjunctive fork, with $B$ being the middle link. But, if forks are to represent causal relations, then $A B C$ cannot be a conjunctive fork, because it is not a fork in the first place. The moral is this: prior causal knowledge is needed to determine whether the fact that a triple of events satisfies conditions (1.3)-(1.6) means that the triple constitutes a conjunctive fork. We differ in this opinion from e.g. Salmon, who considers the statistical conditions as definitional ${ }^{13}$ for the notion of the conjunctive fork, but assumes (for unrelated reasons) that none of the probabilities occurring in the requirements may be equal to 0 or 1 . His reasoning would thus be unaffected by my counterexample.

Conjunctive forks open to the past would of course (just as any examples of two correlated events with neither a common effect nor a common cause) constitute coun-

13 See Salmon (1984), p. 159-160. 
terexamples to the PCC. Reichenbach claims that whenever a conjunctive fork $A E B$ is found such that $E$ is a common effect of $A$ and $B$, there exists an event $D$, which is a common cause of $A$ and $B$, and is the middle link of a conjunctive fork $A D B$. Thus there exist no conjunctive forks open to the past.

Reichenbach offers both a general argument and some specific examples. The argument is of a teleological nature and refers to the fact that we do not accept final causes as explanations. Final causes are deemed incompatible with the second law of thermodynamics in the preceding chapter ( $\$ 18$ of Reichenbach (1971)); a general question is asked: how are we to explain the presence of a highly ordered (and so, very improbable) state of a system (such as a trace of footprints in the sand)? Reichenbach's answer is that we are supposed to look for an interaction "at the lower end of the branch run through by an isolated system which displays order", which will be the cause; "the state of order is the effect" (p. 151). The ordered state is, then, to be understood as a post-interactive state. Since the overarching goal is to provide a definition of time direction (as we have seen Reichenbach doing in the following chapter- 19 of Reichenbach (1971)-by defining what is to be meant by "past"), the author proposes to consider the system containing the beach with the footprints in "reverse time" ( $p$. 153). We would have to think of the ordered state as a pre-interactive state, and so, in our search for its explanation would end up with a final cause ("The wind transforms the molds in the sand into the shapes of human feet in order that they will fit the man's feet when he comes", ibid.); in general, we would "explain the improbable coincidences by their purpose rather than by their cause" (ibid.). Since this is implausible, the conclusion is that the direction of time should be defined, generally speaking, from interaction to order, rather than the other way round. And so, "if we define the direction of time in the usual sense, there is no finality, and only causality is accepted as constituting explanation" (p. 154).

Unfortunately, the nonexistence of conjunctive forks open to the past would follow from the above only had it been established that such a conjunctive fork would necessitate the usage of final causes. This would only be the case if (1) every correlation between events having a common effect but no common cause (i.e. events being the extreme elements of a causal fork open to the past) had an explanation; (2) the only accepted way of explaining such a correlation would be to refer to an event in their causal future. But Reichenbach does not give arguments for any statements similar to the two above; in fact, he seems to rely on an (unsupported) fundamental principle that every correlation whatsoever has an explanation. Notice also the curious jump from the epistemic to ontological perspective on p. 163: "A common effect cannot be regarded as an explanation and thus need not exist". In general, it does not seem that Reichenbach's general argument for the nonexistence of open conjunctive forks with a common effect as the middle element holds up under scrutiny, mainly due to the trick of deriving the ontological conclusion from epistemic premises (like the universal requirement for explanation for correlations). 
Coming now to the specific examples, the author gives an instance of a fork open to the past on p. 163, aiming to convince the Reader that the fork cannot be conjunctive. Let us quote a part of the example:

"For instance, when two trucks going in opposite directions along the highway approach each other, their drivers usually exchange greetings, sometimes by turning their headlights on and off. We have here a fork $A E B$, where $E$ is the exchange of greetings, which is a common effect of the "coincidence" of the trucks, that is, of the events $A$ and $B$ " (Reichenbach (1971), p. 163).

It is not evident how we should think about probabilities in this case, but one way would be to hold fixed a fragment $X$ of some highway, and let $A$ be the event "there is a truck going in the eastern direction in the fragment $X$ ", $B$ be the event "there is a truck going in the western direction in the fragment $X$ ", and $E$ "two trucks going in the opposite directions in the fragment $X$ are flashing their headlights". We can check whether the events occur e.g. every second. Then it is very likely that $E^{\perp}$ does not screen off $A$ from $B$, so the three events indeed do not form a conjunctive fork. Still, a general argument against the mere possibility of such a fork open to the past is needed.

A related problem appears in Salmon (1984), where on p. 164-165 an example offered by Frank Jackson of a conjunctive fork open to the past is discussed.

\begin{abstract}
“[C]onsider a case that involves Hansen's disease (leprosy). One of the traditional ways of dealing with this illness was by segregating its victims in colonies. Suppose that Adams has Hansen's disease $(A)$ and Baker also has it $(B)$. Previous to contracting the disease, Adams and Baker had never lived in proximity to one another, and there is no victim of the disease with whom both had been in contact. We may therefore assume that there is no common cause. Subsequently, however, Adams and Baker are transported to a colony, where both are treated with chaulmoogra oil (the traditional treatment). The fact that both Adams and Baker are in the colony and exposed to chaulmoogra oil is a common effect of the fact that each of them has Hansen's disease. This situation, according to Jackson, constitutes a conjunctive fork $A, E, B$, where we have a common effect $E$, but no common cause" (Salmon (1984), p. 164)
\end{abstract}

To check whether the statistical conditions are satisfied, one has of course to check e.g. probabilities $P\left(A \mid E^{\perp}\right)$ and $P\left(A^{\perp} \mid E^{\perp}\right)$. But how should we do this? We had already assumed that Adams has Hansen's disease and that he is in the colony. How can we ask about the probability that he is not ill or that he is not in the colony? Certainly we are not evaluating a probability of a counterfactual statement ${ }^{14}$. Instead, it is evident from p. 165 of Salmon (1984) that the author calculates the probability $P\left(B^{\perp} \mid E\right)$ simply by taking the proportion of people in the colony who are not ill (the medical personnel) to all members of the colony. But in this way he transforms a constant into

14 Which is a task attempted later on e.g. in chapter 7 of Pearl (2000). 
a variable and it is no longer possible to differentiate between events $A$ and $B$, since both of them are "a randomly chosen man from the colony has Hansen's disease".

It would seem, then, that Reichenbach's account lacks a general argument for his point, and Salmon's considerations on the subject are defective. On the other hand, we have to admit we have been unable to find a "real-world" example of a conjunctive fork open to the (causal) past. Still, consider the following hypothetical situation: a group of 10000 men (labeled, for our convenience, from 1 to 10000) considered as representative for the region is tested for hypocalcemia $(E)$, lactose intolerance $(A)$ and hypoparathyroidism $(B)$. Lactose intolerance and hypoparathyroidism have no known common cause, while it is known that each may lead to hypocalcemia. If:

- men labeled from 1 to 1000 (and only them) have hypocalcemia;

- men labeled from 1 to 500 and from 1001 to 4000 (and only them) have lactose intolerance;

- men labeled from 251 to 750 and from 3001 to 6000 (and only them) have hypoparathyroidism;

then it is straightforward to see (if we accept the move from relative frequencies to probability: here, for the sake of the example, we can simply say that the population from which the sample had been drawn is identical to the sample) that the fork $A E B$ satisfies the requirements from the definition of a conjunctive fork (1.3)-(1.6). However, the middle element of the fork is a common effect of the two other elements, which have no known common cause. I do not see why such situations should be impossible; yet again, I have been unable to find a "real" example. ${ }^{15}$

(A different matter is whether a "conjunctive fork open to the past" and a "conjunctive fork $A E B$ with the middle element $E$ being a common effect of $A$ and $B$, such that there is no common cause $C$ creating a conjunctive fork $A C B$ " are to be identified. They certainly are on the assumption that "past" in the first expression is to be understood as “causal past”).

\subsection{The PCC After Reichenbach}

Reichenbach's Principle was heavily promoted in the 70s and 80 s by Wesley Salmon (e.g. Salmon (1971)). More recently, it has been an inspiration for a fundamental condition in the field of representing causal relations by means of directed acyclic graphs (see chapter 4). However, a plethora of counterarguments appeared; most are gathered and discussed in Arntzenius (1992). Some, e.g. Sober's (1988) "sea levels vs. bread prices” argument, were directed against any sort of general requirement of

15 Another hypothetical example of a conjunctive fork not pointing to a common cause was also presented in Torretti (1987). 
common causal explanation. Others led a few philosophers (e.g. Salmon (1998b) ${ }^{16}$ and Cartwright (1988)) to transform Reichenbach's idea, preserving the Principle's requirement of common causes for correlations, but changing the screening off condition, or supplementing it with other conditions. It would be of no use for the current essay to discuss all these ideas in detail: our focus is on the notions of common cause revolving around the original idea of screening off. We will however describe the three arguments we would rate as most important. These are:

- the argument from Bell inequalities, to which we will devote the whole chapter 3;

- the argument from conservation principles, described in section 1.4.1;

- $\quad$ and the "sea levels vs. bread prices" argument, described in section 1.4.2.

Later, starting in the 90s, Reichenbach's idea in the form of PCC 4 was defended in papers by M. Rédei, G. Hofer-Szabó and L. Szabó (e.g. Hofer-Szabó et al. (2000)): rather than confronting the earlier counterarguments to Reichenbach's idea directly, the authors proposed mathematical arguments in favour of PCC 4. It is to this area of research that the current study aims to contribute in chapters 5 and 6 . Let us first describe the two arguments against Reichenbach's Principle we just mentioned above.

\subsubsection{The Argument from Conservation Principles}

We will cite the formulation of this argument given in Arntzenius (1992), since it seems to be the most concise: ${ }^{17}$

\footnotetext{
"Suppose that a particle decays into 2 parts, that conservation of total momentum obtains, and that it is not determined by the prior state of the particle what the momentum of each part will be after the decay. By conservation, the momentum of one part will be determined by the momentum of the other part. By indeterminism, the prior state of the particle will not determine what the momenta of each part will be after the decay. Thus there is no prior screener off." (Arntzenius (1992), p. 227-8.)
}

There are numerous variants of this argument in the literature; the version from Salmon (1998b) refers to Compton scattering. In the same paper Salmon, as an answer to the problem, proposes the introduction of another kind of fork (apart from the conjunctive variety), the so called interactive fork. Probabilistically, an interactive fork with the middle element $C$ and two extreme elements $A$ and $B$ differs from a corresponding conjunctive fork in that instead of the two screening off requirements a single condition is introduced: namely, $P(A B \mid C)>P(A \mid C) P(B \mid C)$. That it is met by

16 Originally published in 1978.

17 It is labeled "Indeterministic Decay with Conservation of Momentum" and attributed to van Fraassen (1980). 
the examples built around some conservation principle becomes evident when we notice that in such examples (if $C$ is the state of the compound before the splitting) $1=P(A \mid B \wedge C)>P(A \mid C)$.

Notice that the argument only implicitly refers to probability, via the notion of screener-off. No probability spaces are defined. Therefore it is an argument against PCC 2. It is not clear what force it would have against PCC 4, which-as mentioned above-has been mathematically proven to be true. One would have to consider all probability spaces which could be used to describe the decay event and its consequences; then the extensions of those spaces which contain common causes in the sense of PCC 3; and finally ponder the question whether such events could have anything to do with what we would naturally accept as a common cause of the properties of the two particles.

An ad hoc solution-but not without intuitive merit-on part of a proponent of PCC 2 could be that correlations which arise due to conservation principles do not demand (additional) explanation: if we know the principle at work, we do not require anything more to explain the correlation. Salmon's way out (philosophically rooted in the distinction between causal processes and interactions) was simply to incorporate interactive forks into the picture and to say that some correlations are explained by events which together with the correlated events form a conjunctive fork, but some others demand as their explanantes the middle elements of interactive forks. ${ }^{18}$

\title{
1.4.2 The "Sea Levels vs. Bread Prices" Argument
}

This argument first appeared in Sober (1988) and was elaborated in Sober (2001). The most important thing is that, in the parlance of the current essay, it is an argument for abandoning PCC 1 for PCC 2: not all correlations demand a common causal explanation. ${ }^{19}$ By reductio: otherwise a correlation between Venetian sea levels and British bread prices would demand such an explanation, while it surely does not. More extensively:

\begin{abstract}
"Consider the fact that the sea level in Venice and the cost of bread in Britain have both been on the rise in the past two centuries. Both, let us suppose, have monotonically increased. Imagine that we put this data in the form of a chronological list; for each date, we list the Venetian sea level and the going price of British bread. Because both quantities have increased steadily with time, it is true that higher than average sea levels tend to be associated with higher than average bread prices. The two quantities are very strongly positively correlated.
\end{abstract}

18 In fact, Salmon himself eventually espoused a variant of the "conserved quantity" theory, see Salmon (1998a).

19 Which demand and which do not is in Sober's account decided by our background theory. 
I take it that we do not feel driven to explain this correlation by postulating a common cause. Rather, we regard Venetian sea levels and British bread prices as both increasing for somewhat isolated endogenous reasons.” (Sober (1988), p. 215.)

There are several strands of thought in the literature on the argument. We will try to label and shortly discuss them.

1. No correlation at the level of changes. Forster (1988) was the first to notice that, while the sea levels and bread prices are correlated, their respective changes are not: year by year, both the former and the latter increase. It is hard to estimate the import of this observation: in fact, the lack of correlation on the level of changes would perhaps intuitively indicate lack of causal connection, which would strengthen Sober's point. In any case, Sober (2001) presented an example stemming from evolutionary biology in which the correlation persists on the level of changes of the values of two attributes, and in which also no common causal explanation is expected.

2. Mixing. Some authors (e.g. Spirtes et al. (2000), p. 33-37) point out-going back to the work of G.U. Yule in the beginning of the XXth century-that a correlation between attributes in a population may be the result of mixing two populations in which the attributes are not correlated. The solution of Spirtes, Glymour and Scheines, working in the formalism of directed acyclic causal graphs (more on that in chapter 4), is to treat "belonging to one of the given subpopulations" as an attribute in the "big" population, which enables them to recover the proper conditional independencies. This amounts to saying that whenever such a mixing occurs, the correlation is explained by the mixing itself. The authors treat ( $\mathrm{p}$. 37) Sober's example as a case of mixing (in fact, they claim that his point was similar to Yule's) and consider the case closed. This is consistent with PCC 2: if we know that such a mixing occurred, then we either do not think of the correlation as demanding an explanation at all, or we consider it explained by the mixing itself. However, the particular example of sea levels and bread prices does not lend itself easily to the "mixing" interpretation. What are the two populations to be mixed? They cannot be the 200 years, since there is only one set of years to be considered. Thinking of Sober's example as a case of mixing seems to require some serious mind-twisting. It might be better to look at it as consisting of two monotonically increasing time series.

3. Time series. Hoover (2003) observes that the data given by Sober allow us to infer that there is a correlation on the level of frequencies, but it does not necessarily follow from that that there is a correlation on the level of probabilities. "(...) most statistical inference and most of our own probabilistic intuitions are based on stationary probability distributions” (p. 532).

A rigorous discussion of this point would require numerous definitions, so let us settle for a more informal account. A necessary condition for a time series to be stationary is that "the covariance between the values of the series at different 
times depends only on the temporal distance between them” (p. 532). Of course, a monotonically increasing time series is not stationary. However, for any time series we can consider a series of differences between the consecutive values of the series. It may very well happen that the series is stationary (consider e.g. the case of the series of consecutive natural numbers from 1 to 200); in that case the original series is said to be integrated of order 1, or "I(1)". As Hoover points out (p. 545-546), a linear combination of two I(1) time series is in general also I(1), but it may happen that there exists a linear combination which is stationary: only in this case (in which we say the time series are cointegrated) the data constitute evidence for probabilistic dependence. As Reiss (2007) puts it (p. 184), “inferring from a sample correlation to a probabilistic dependence means that one takes the most likely data-generating process to be stationary”. In Sober's case we are likely to assume otherwise. Hoover proposes to let the PCC apply to cases in which the correlated series are either (1) both stationary or (2) both I(1), but cointegrated. Since Sober's time series are not cointegrated, they do not constitute a counterexample to Hoover's version of the principle.

The general direction of these replies against the "sea levels vs. bread prices" argument seems to be that, while Sober's paper could be interpreted as containing a probability space with unexplained correlations, it is doubtful whether that space corresponds to anything "in the world"; whether there really are factors correlated in the way the probability space would have it. Inspection of a mathematical object, even a probability space constructed on the basis of actual, observed frequencies, is not enough to disprove the Common Cause Principle in its PCC 1 formulation.

We would like to divert attention to a different issue. Why do we think the correlation between Venetian sea levels and British bread prices does not demand explanation? Is there more to say on the topic than Hoover's idea of referring to the two time series being nonstationary but not cointegrated?

\subsubsection{Which Correlations Demand Explanation?}

Various authors have fleshed out the beginning part of PCC 2 differently. Some say that only "improbable" correlations demand explanation. This can mean simply "statistically significant” (Forster (1988), p. 539), “unexpected or surprising” (Uffink (1999)), or "such that the assumption that it arises from causally unrelated processes will render it unexpected", with a formally defined notion of "unexpected” (Berkovitz (2000), p. 65).

There is a sense, meta-probabilistic in a way, in which a correlation can be rigorously thought of as "improbable". Consider a finite probability space $\langle\Omega, \mathcal{F}, P\rangle$, in which $\Omega$ has $n$ elements, $\mathcal{F}$ is the set of all subsets of $\Omega$, and for any $A \in \mathcal{F}, P(A)=$ $\frac{\operatorname{card}(A)}{n}$. Suppose two subsets $A$ and $B$ of $\Omega$ are chosen at random in the sense that every 
member of $\Omega$ has a chance of $\frac{1}{2}$ of belonging to $A$ and the same chance of belonging to $B$. It can be checked using a moderate dose of combinatorics and Stirling's approximation that with $n$ approaching infinity, the chance of arriving at a probabilistically independent pair by the process just outlined approaches 0 . Informally, we would say that "in a big enough population, (almost) everything is correlated with (almost) everything". But the proportion of pairs such that $|P(A B)-P(A) P(B)|<0.05$ (which is one, quite arbitrary, way of saying "weakly correlated pairs") to all pairs increases with $n$, too-strongly correlated pairs are infrequent in this sense (i.e., it is hard to come upon them by pure chance). (I have to confess that for this statement I only have an argument of "consulting statistical software"-having used the " $R$ " software ${ }^{20}$ to track the proportions of weakly and strongly correlated pairs in populations of increasing sizes.) So, if we do not possess any knowledge about the genesis of some two events, it should be natural to expect them to be correlated, but only weakly. The subjective degree of this expectation should vary with the size of the population involved. However, this approach is too abstract to properly illustrate our beliefs regarding the probabilistic nature of phenomena we observe in the world: we in general do not start with a "clean slate", but possess some background knowledge which influence our beliefs regarding such issues.

In his account ${ }^{21}$, to the condition of "improbability" Berkovitz also adds, without further commentary, that the correlation should be "non-accidental" (p. 56). What does it mean for a correlation to be accidental? Perhaps it will be illuminating to consult a clarification of types of correlation from Haig (2003), where a reinterpretation of the notion of spuriousness is argued for.

A traditional (see e.g. Hitchcock (2010)) example of a spurious correlation is that of a barometer and a storm. Shortly speaking, a spurious correlation arises between events which are not directly causally connected, but have a common cause. Haig notices that the word "spurious" might be misleading in this case, since the correlation is due to a genuine causal connection-in contrast with the correlations which arise e.g. from a sampling bias. Haig's classification is presented in figure 1.2.

A "nonsense correlation" is that for which "no sensible, natural causal interpretation can be provided" (Haig (2003), p. 127). Haig's examples are "the high positive correlation between birth rate and number of storks for a period in Britain" and "the negative correlation between birth rate and road fatalities in Europe over a number of years". It is clear, I think, that Haig would interpret Sober's examples as falling into this category. "Spurious correlation" are these which are "not brought about by their claimed natural causes", but "by accident", for example due to "sample selection bias, use of an inappropriate correlation coefficient, large sample size, or errors of sampling, measurement, and computation” (Haig (2003), p. 128). Of the genuine cor-

20 R Core Team (2012).

21 Berkovitz (2000). 


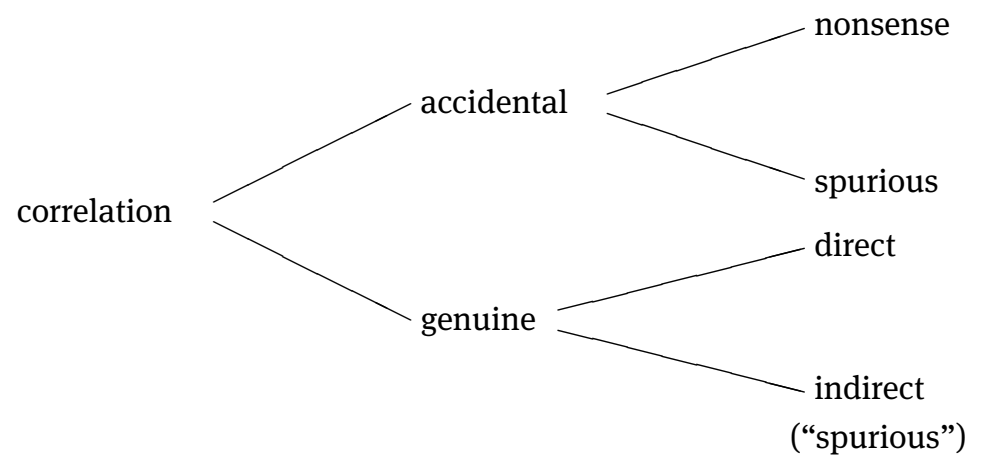

Figure 1.2: A classification of correlations from Haig (2003).

relations, the "indirect" are due to either "common or intervening causes" (p. 129)22. Notice that if we formulate our Principle as "any non-accidental correlation between events which are not directly causally related or logically dependent is due to a common cause" it is clearly true under Haig's account. Whether the common cause in question should screen off the correlated events is a different matter.

On the other hand, Sober would most likely say that-in the cases like the "sea levels vs. bread prices"-there is a perfectly natural causal interpretation which consists of two separate causal explanations of the two phenomena, and thus such correlations should not be counted as "nonsense". But let us examine this line of reasoning. Suppose that there is an explanation $E$ for the ongoing rise of sea levels; perhaps the melting of sub-polar glaciers. $E$ explains why the data for sea levels in Venice form a monotonic time series. Likewise, suppose there is some explanation $F$ for the ongoing rise of bread prices; perhaps a combination of high taxes and deteriorating crop levels. $F$ explains why the data for bread prices in Britain form a monotonic time series. But this does not yet explain the correlation in question. But why is $A$ higher than average in the given time period precisely whenever $B$ is? The only reason we can find is that the processes $E$ and $F$ are active in the same time period. If this is accidental, then the correlation between $A$ and $B$ should be deemed as accidental, too. Van Fraassen (1991, p. 350) uses the word "coincidence" in this context.

22 A correlation between $X$ and $Y$ can arise partially due to an intervening cause if there is a cause $Z$ which with $X$ jointly produces $Y$. 


\subsection{An Epistemic Position}

It is interesting that in recent years two diametrically opposed approaches to the PCC have emerged: one (by Rédei, Hofer-Szabó and Szabó, discussed in chapter 6) we could label as "maximalist" aiming to prove that any correlation has an explanation by means of a common cause if the probability space is suitably chosen, and one perhaps aptly labeled as "minimalist”, due to Reiss (2007). The latter renders the PCC as an intrinsically epistemic principle. Since it seems to have an illustrative purpose and amounts to saying "if there is no conflicting knowledge, correlation is evidence for causation", we will only give the formulation here without extensive discussion:

PCC(Reiss). "The proposition $e=$ "Random variables $X$ and $Y$ are (sample or empirically) correlated" is prima facie evidence for the hypothesis $h=$ " $X$ and $Y$ are causally connected". If all alternative hypotheses $h_{i}^{a}$ (e.g. "the correlation is due to sampling error", "the correlation is due to the data-generating processes for $X$ and $Y$ being non-stationary, " $X$ and $Y$ are logically, conceptually or mathematically related”) can be ruled out, then $e$ is genuine evidence for $h$ ” (Reiss (2007), p. 193).

The epistemic nature of the Principle is evident from its use of the notion of "evidence"-no existential statements about the world are to be inferred from other statements of a similar nature; the Principle concerns the shaping of our conception of the causal structure of the world. The Principle is eminently fallible because we may simply have wrong evidence against the hypotheses alternative to the one of the existence of a common cause.

Before we tackle the issue of Bell inequalities, it is fitting to devote a chapter to the formal features of various notions related to screening off. 